\title{
Study on Consumer Behavior Predict in E-commerce Based on Multi-Agent
}

\author{
YanRong Zhang ${ }^{1}$ and ZhiJie Zhao ${ }^{1}$ \\ ${ }^{1}$ School of computer and information engineering \\ Harbin University of Commerce \\ Harbin, China \\ zhangyanrong_5@163.com
}

\begin{abstract}
This paper adopted the method of user interest concept tree based on domain ontology, and proposed a multi-agent based consumer behavior forecasting model in e-commerce to overcome the limitations of traditional consumer behavior forecasting method. The model consists of multiple agents. They are consumer behavior forecast agent, user interest model management agent, monitoring agent and source data monitoring agent. These agents collaborate with each other and then predict consumer behavior. In order to validate the feasibility of the model, the paper combined with the artificial intelligence technology, the database technology, the networkings as well as the electronic commerce correlation technique to build e-commerce consumer behavior predict expert system. From the test results, compared with the original method, it can effectively analyze and predict e-commerce customers consumer behavior, and can be concluded that the customer's overall consumption trend.
\end{abstract}

Keywords: E-commerce, Consumer Behavior, Multi-agent

\section{Introduction}

With the rapid development of Internet, e-commerce has developed rapidly. The Internet is increasingly becoming an integral part of our lives, online shopping is becoming a new shopping channel. Many enterprises and businesses have begun to catch the development trend of information and each established their own websites and forums, and on the basis of the traditional business model, the use of Internet technologies, open e-business model, renewal and modernization of enterprises marketing and strategic development of the original model. In increasingly competitive time, in order to get the survival and development of enterprises under e-commerce, consumers are key factors and consumer behavior to a certain extent on enterprise development with the overall pattern will have a big impact. Therefore, understanding of consumer behavior and information behavior will no doubt provide them with goods and services, greater relevance, thereby improving the quality under the environment of e-commerce sales and customer services, thereby enhancing the competitiveness of online businesses.

The paper according to the experience of E-commerce expert, based on the agent technology, under the Web environment, take Sun JDK 5, Eclipse 3.1, Dreamweaver and so on as the development environment, uses the B/S structure and based on the J2EE standard development pattern, has constructed an Consumer Behavior Predict Expert System of Ecommerce using Java language. It provides the product information of consumers interested. This improves efficiency and effectiveness in running e-commerce platform in the Internet environment, thereby improving the quality of online businesses, sales and customer service, 
enhance the competitiveness of online businesses. E-commerce Web sites, understanding consumer behavior characteristics of shopping behaviors and information on the site, will no doubt provide them with goods and services leads to greater targeted; conducive to shopping sites to Web site organization, effective use of information content and management, improve the quality under the environment of e-commerce sales and customer services, thereby enhancing the competitiveness of online businesses has the vital significance.

\section{Agent and Expert System}

\subsection{Agent and Fundamental Model of Agent}

2.1.1. The Definition and Characteristic of Agent: Agent is one kind of computation entity or procedure. It is able to perceive the environment under the specific environment, and be able to run Autonomy to realize a series of goal, the goal of its designer and the user, its principal characteristic has the presence, the independency, the reactivity, the initiative and the sociality. Multi-Agent (MAS) is composed of independent or half independent intelligent body, its development for the solution complex question decision-making, established distributional, intellectualized, the integrated man-machine harmony decision support system to provide the new method and means, its important characteristic had the sociality, the selfrestraint and the cooperation. In view of the above agent characteristic, applies the agent technology in the consumer behavior predict expert system, may increase Customer behavior intellectualization and personalized processing [1].

2.1.2. Expert System: According to people's understanding, expert system is defined as: "An expert system is a design used to problem-solving capabilities of human experts, modeling computer program." An expert system is an intelligent computer program system, the internal of expert system contains a large number of knowledge and experience of the level of experts in one field, can take advantage of human expert knowledge and problem-solving approach to deal with the problem in this area. In other words, the expert system is a great deal of specialized knowledge with experience in program system, which use artificial intelligence and computer technology, according to a certain area of one or more of the expert knowledge and experience, reasoning and judgments, simulation of human experts decision-making process in order to address those of human experts to deal with the issue of a hybrid. In short, the expert system is a simulation of the field of human experts to solve the problem of a computer program system [2].

\section{Consumer Behavior in e-commerce}

Consumer behavior refers to meet the needs and desires of consumers looking for evaluation, select, purchase, use, and disposal of the products and services of the activities and processes involved. While the consumer behavior in electronic commerce refers to the consumer in the sale and purchase of goods and services on the Internet, shopping information through computer networks to handle business transactions with the funds transfer.

Under the various factors (including individual factors, environmental factors and marketing factors), Consumers come into being a certain sense of self and their lifestyle, specific self-consciousness and lifestyle can lead to appropriate consumer needs and motivations. Consequently, this correspond the consumer behavior. And the realization of consumer behavior will impact the consumer characteristics of internal and the external environment. 


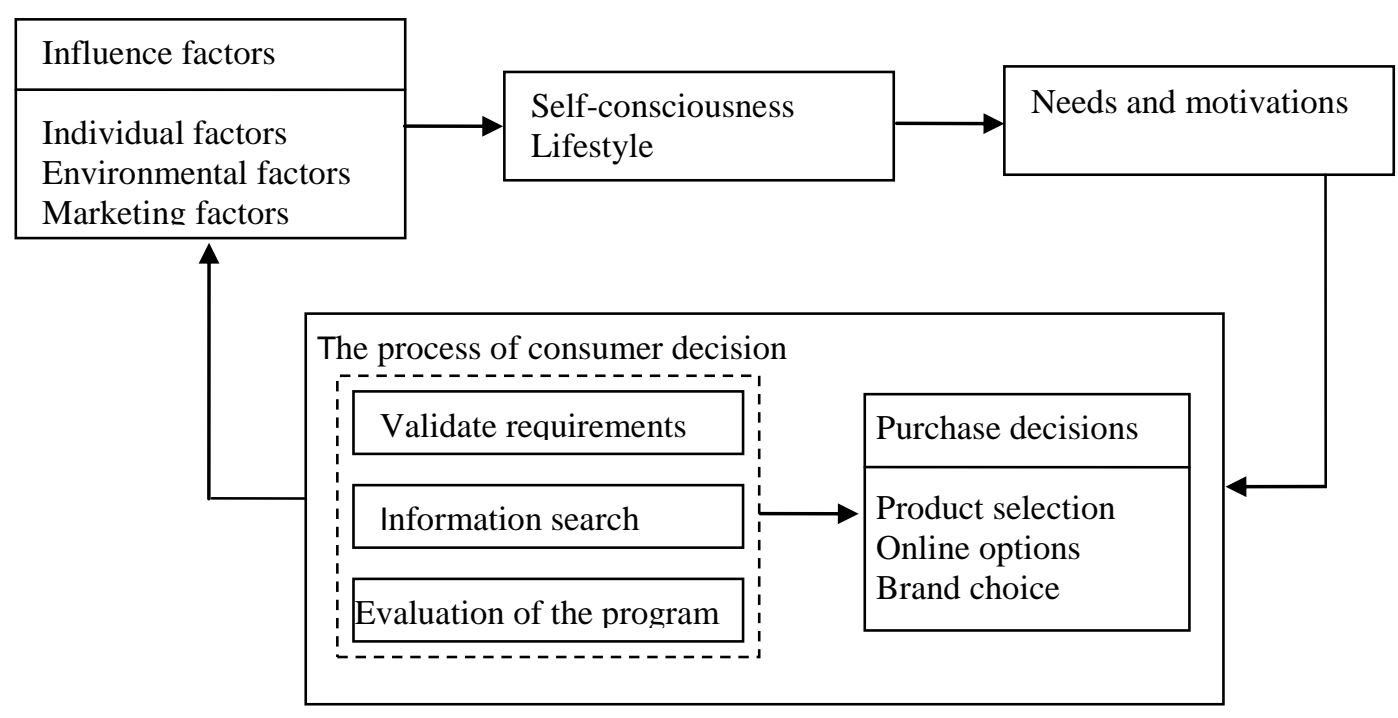

Figure 1. The Analysis Model of Consumer Behavior in E-commerce

\section{Consumer Interest Model}

Building user interests model is the prerequisite and basis for prediction of the consumer behavior in electronic commerce. Only created user profile could proceed ad matching. It applies agent technology to identify the project, which matches the user model. Before ecommerce consumer behavior can be predicted. So, consumer interest model is the foundation of consumer behavior forecast.

\subsection{The Access of Consumer Interest Information}

The information of e-commerce consumers interested can be obtained in the following seven ways [3]:

(1)User's personal information that he selected or inputted, such as occupation, age, sex, topics of interest and other information.

(2) The bookmarks that user saved.

(3) The documents that user browsed.

(4) Behavior of the user browsing the page, including copying, printing, scrolling, clicks of the mouse, and user presence time.

(5)The documents that user saved and downloaded.

(6) The log information of server saved.

(7) The keywords that user queried.

In this consumer behavior predict expert system, early users are getting user information by the user manually input their ages, professions, occupations and other information, and from the drop-down menu, select your favorite categories of interest. After the user starts using the system, primarily through the hidden ways to get users ' browsing behaviors, mining what users are interested in information through user behavior [4]. 


\subsection{Consumer Interest Model Representation}

In this paper, we use the Representation method of ontology to describe the consumer interest model. Based on an ontology building user interests' model is often used to represent the information of interest to the user. General topics of interest to the user level in the form of display, each node represents a user's interest in the subject. Ontology is able to convey the complex relationships between concepts; users expressed interest in complex information. The semantic relations in the information of users ' needs into the main body of the relationships between nodes, this method can express the users ' needs truly.

This consumer interest model representation adopted the approach of consumer interest concept-tree based on domain-specific ontology. In this consumer interest concept tree, each node is defined as a triple, and each node is composed of three parts: node $=\{$ keyword, weight, time $\}$. Among them, keyword is representing the interest topics of the nodes, weight is the weight value of the interest topics, and enjoying on behalf of a user interested in this topic. Within its range in real numbers, the larger, representative user more interested on the topics of interest, on the contrary, the smaller the number, it means the user is not interested in. time was the subject that interest the weight value on the last updated time [5].

Interesting concept-tree generation algorithm based on domain ontology is as follows:

(1)The user account is set as an interesting concept-tree root node, it is to identify users uniquely identify that represents the user's personal information.

(2) Based on user interest concept $k$ creates user interest child nodes in the tree, reference to the relationship between interest in domain ontology classes and find the concept corresponds to the parent of child nodes; user interest concept tree, child node from the parent node that corresponds to the connection, the parent node and the root node to connect users ' personal information.

(3) Calculate weighted values for child $k$. The weight value of $\mathrm{k}$ is subject to the child node under which all of the weighting value of the feature word node $t_{i}$ and coupled with the weight of its own value, as in (1). Among them, the calculation of weighting value of the features word node $t_{i}$, as in (2). Where $t_{j} f_{j}$ is the number of occurrences of characteristic word. $g$ is the corresponding weighting factor.

$$
\begin{gathered}
\text { k.weight }=\sum_{i=1}^{n} t_{i} \cdot \text { weight }+k . \text { weight } \\
t_{i} \cdot \text { weight }=\sum_{j=1}^{3} t_{i} f_{j} \times g_{j}
\end{gathered}
$$

(4)Calculate the weight value of the parent node. The weight value of the parent node is the sum of all child nodes' weight values, as in (3).

$$
\text { p.weight }=\sum_{i=1}^{n} k_{i} \cdot \text { weight }
$$


Consumer interest modeling using automated user modeling. Automated user modeling is that system based on user behavior and should be able to view the content, such as page dwell time, add to Favorites, print the document, and automatically build user models [6].

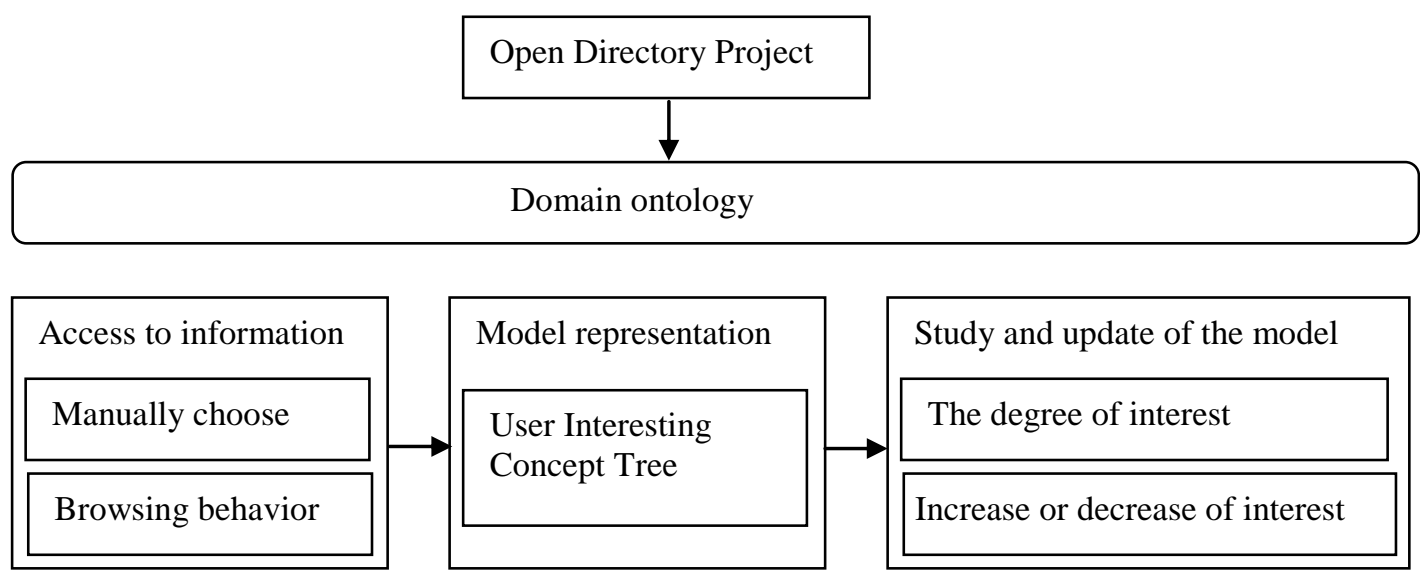

Figure 2. The Establishment of User Interest Model

\section{Consumption Forecasting Model based on Agent}

This paper proposes a multi-agent-based e-commerce forecasting model of consumer behavior. Its design is done using agent technology will be the feature model for composed of multiple agent. Among them, the core for consumer behavior forecast agent (CBFA). User interest model management agent (UIMMA) is the premise and foundation of consumer behavior forecast. Monitoring agent (MA) for agent trigger consumer behavior prediction, source data monitoring agent (SDMA) is consumer behavior forecast for passing information to the agent, agent evaluation feedback, data mining interest management agent, the user agent is a user agent provides services. Interaction between them, work together on consumer behavior forecast. It's composed of various modules of functionality to the model below for details and design ideas. Overall architecture is as show in Figure 3.

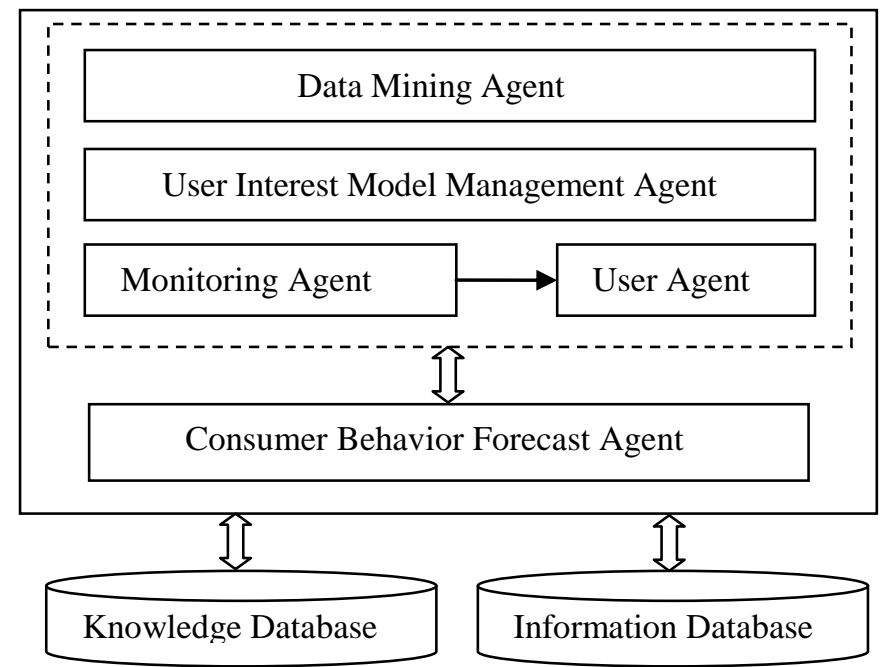

Figure 3. The Basic Model of Consumption Forecasting based on Agent 


\subsection{User Interest Model Management Agent}

User interest model management agent completed the build and update of user interest modeling. User interest model building is the premise and basis of individualized services. A perfect user interest model can adequately reflect the user's real needs, to serve as a basis for prediction of consumer behavior to get good results. With regard to user interest model, user interest is oriented to user needs mining collection, and it is available for users interested in changing user information updates.

\subsection{Data Mining Agent}

Data mining agent's main duties is autonomous to accept recommended engine agent of requests, through web log mining, and decision-making tree, and associated rules, and return algorithm, and neural network, method, for looked purchased acts and access acts of analysis, gets user of needs and interest hobby, auxiliary user interest model management agent completed task; while produces looked access products sequence in the of associated rules, and customer classification, and similar customer groups and similar customer evaluation forecast, mode, provide the basis for consumption forecasting Agent [7].

\subsection{Monitoring Agent}

Monitoring agent's primary responsibilities is to monitor the actions of users on the userinterface agent and browsing behavior. Monitoring agent to capture customer information for logging into e-commerce site, and on the basis of the customer's browsing or buying consumer behavior-prediction Agent sends a request. Monitoring agent can be either active or passive means of implementation. Own ways is the captured Agent looked in the user interface-related behavior, prediction Agent informed consumer behavior to predict customer buying behavior. Passive customers can interact through a user interface agent and Monitoring agent, search product requirements from users to monitoring agent, by its circular consumption forecast to predict consumer behavior for the user agent. Also, monitoring agent also will timely to captured user browse acts or purchased acts of changes, once looked of acts and interest occurred changes, it will timely to user interest model management agent, and data mining agent notices corresponding of information changes, user interest model management agent, and data mining agent can timely to on user of acts for analysis, timely update user interest model, for consumption acts forecast agent provides latest of user information and interest hobby.

\subsection{Consumer Behavior Forecast Agent}

Consumer behavior forecast agent is the core of consumer behavior predicting system. This paper will be designed as a multi agent system. They are search agent, control Agent and results generated agent.

Search agent's task is for accepting consumer behavior forecast agent's requests, searchrelated information from the repository, providing information for consumer behavior forecast agent collection.

Main function of control agent is that when the data source has more changes, Control agent monitoring agent receives the source database reports, changed data involves the judgment of the model, command active mining data mining agent, management agent communicates with the user profile, to dynamically update user profile.

Results generated agent Based on user interest model and user database, using computer algorithms for predictive results will be returned to the system. 


\section{Consumer Behavior Predict Expert System's Designs and Realization}

The overall system uses the B/S structure. The Web server uses Tomcat, the client side user may visit this system through any Web browser. The management, memory and maintenance of knowledge library uses Oracle $10 \mathrm{~g}$ database to realize. Realizes using the JSP technology to visit the knowledge library and design the inducing equipment [11]. In the system knowledge library there saved more than 1000 kinds of basic information and picture information of goods. The interface of consumer behavior predicts expert system of Ecommerce is as shown in Figure.4.

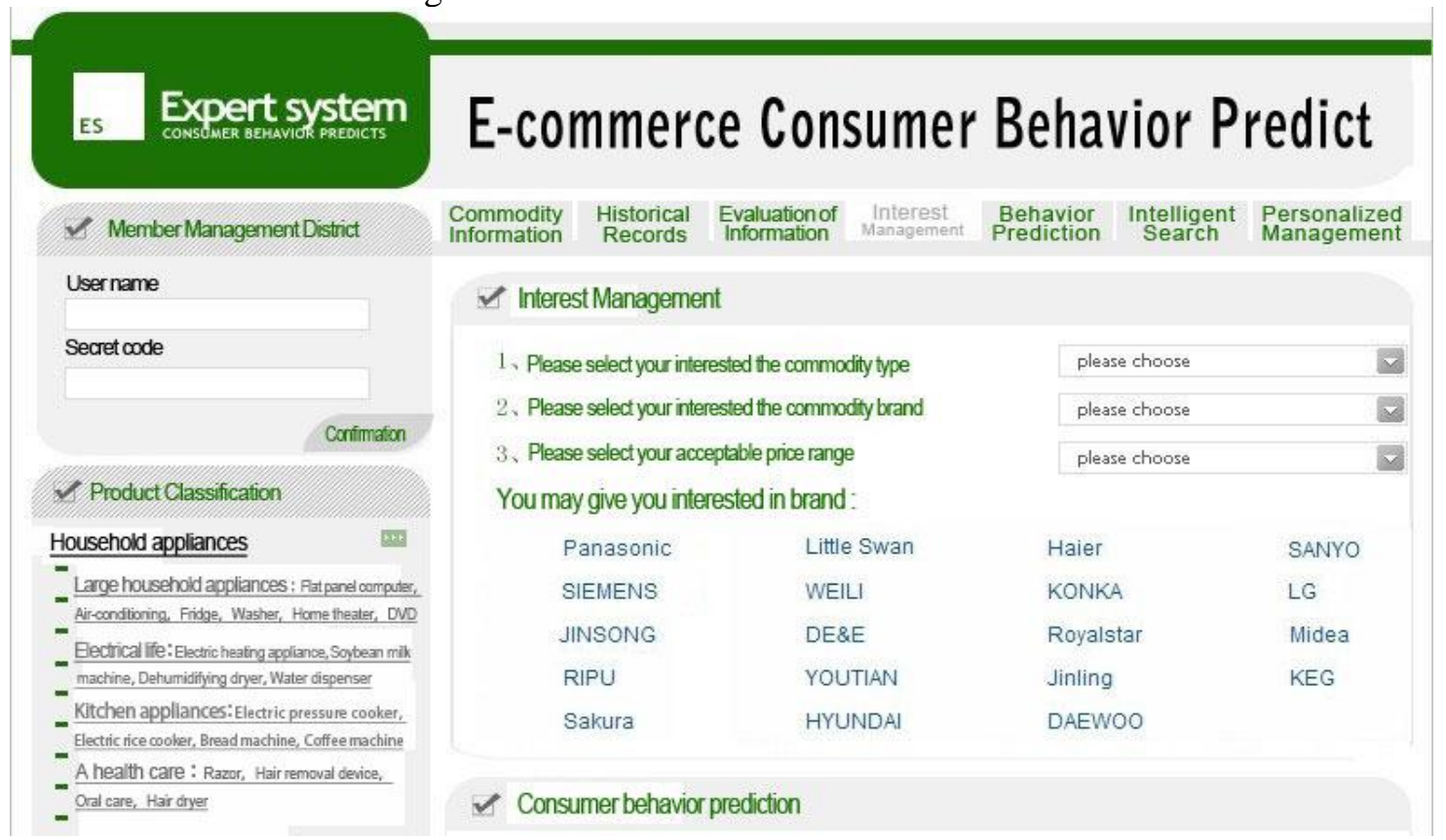

Figure 4. The Interface of Consumer Behavior Predict

Consumer behavior predicts expert system based mainly on different users ' online purchases, recommendation algorithms to get customers preferences and commodity association rules, after the user logs on to the system, system to predict the user behavior. In this system, each registered user has a user interested in description, if a purchase history, historical records and correspondence, these projections provide the basis for a system of information. Unregistered user browsing behavior will be converted into a group of users browse the interest saved by the system, this provides a reference for the user consumption forecast.

When using the system, monitor agent take the initiative to monitor and record customer login, and track the browsing behavior of customers in real time, notifying the user interested in model management agent and consumer behavior forecast agent preferences analysis and consumer behavior. Monitoring agent to keep perceived status and changes in the external environment, timely response to influence the external environment allows the system to take the initiative at the right time to take proactive action. System with independent learning ability, and has a certain reasoning ability, makes it more accurate to speculate on the user's intent. Systems through multiple agents working together can analyses the complex task and come up with appropriate solutions for problems. 


\section{Conclusion}

This system uses the knowledge engineering method under the instruction of system engineering thought, makes the Consumer Behavior Predict Expert's knowledge, experiences and the method of solve question systematization and formalization. Agent technology, the artificial intelligence technology, the database technology, the networking as well as Ecommerce correlation technique studies is applied in the system. A set of advanced and useful expert system is established.

The system can analyze customer behavior and understanding consumer behavior characteristics and take effective steps to guide the consumer behavior. With using the system, e-commerce enterprises to conduct business activities, thereby enhancing the enterprises ' market competitiveness. It has certain theory and practice significance to Ecommerce information technology's promoted application.

\section{Acknowledgements}

This work was supported by Educational Science and Technique Research Program of Heilongjiang Province (Research on E-commerce consumer behavior predict based on rough set theory) and Doctoral Scientific research Project of Harbin University of Commerce (Analysis of e-commerce consumer behavior and its influencing factors).

\section{References}

[1] V. R. Komma, P. K. Jain and N. K. Mehta, "J. An approach for agent modeling in manufacturing on JADE"M reactive architecture", Int. J. Adv. Manuf. Technol., (2011), pp. 1079-1090.

[2] W. D. Potter, X. Deng, J. Li, M. Xu, Y. Wei, I. Lappas, M. J. Twery and D. J. Bennett, "Aweb-based expert system for gypsymoth risk assessment [J]", Computers and Electronics in Agriculture, vol. 27, nos. 1-3, (2000), pp. 95-105.

[3] F. Abbattista, M. Degemmis P. Licehelli, G. Semeraro and F. Zambetta, "Improving the Usability of an Ecommerce website through Personalization", In proceedings of the workshop on Reeommendation and Personalization in Ecommerce, (2002).

[4] R. J. Kuo, J. L. Liao and C. Tu, "Integration of ART2 neural network and genetic k- means algorithm for analyzing webbrowsing Paths in eleetronic commeree", Decision Support Systems, vol. 40, (2005), pp. 355374.

[5] D. Zhang and W. S. Lee, "A Web2based Question Answering System [A]", In Proceedings of the SMA Annual Symposium 2003, NUS, Singapore, (2003) January.

[6] Y. Li and D. Cui, "Improvement and application of MVC design patterns [J]", Jisuanji Gongcheng/Computer Engineering. (2006), pp. 62-64.

[7] R. M. Muzika, S. T. Grushecky and A. M. Liebhold, et al. "Using thinning as a management tool for gypsy moth: the influence on small mammal abundance [J]", Forest Ecology and Management, vol. 192, nos. 2-3, (2004), pp. 349-359.

[8] R. D. Burke, K. J. Hammond and V. Kulyukin, et al. "Question answering from frequently asked question files: ex-periences with the FAQ finder system [J]”, AI Magazine, vol. 18, (1997), pp. 57-66.

[9] V. Hatzivassiloglou, J. L. Klavans and M. L. Holcombe, et al. "SIMFINDER: A flexible clustering tool for summa-rization [A]", NAACL Workshop on Automatic Summarization [C], [s. 1.]: Association for Computational Linguistics, (2001).

[10] R. Schank, "Identification of conceptualizations underlying natural language", Computer Models of Thought and Language, (1973).

[11] Roger's Pressman, Software Engineering: A Practitioner's Approach, WCB McGraw-Hill, (2002). 


\section{Authors}

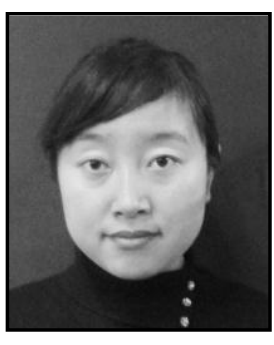

Yanrong Zhang, Doctor, Lecturer, teacher of School of the Computer and Information Engineering, Harbin University of Commerce. Her main research fields are E-commerce and Artificial Intelligence.

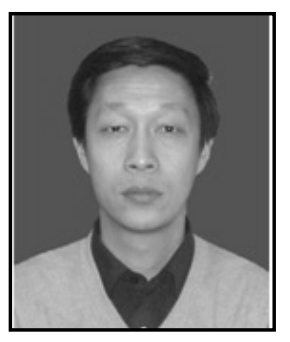

Zhijie Zhao, Doctor, Professor, IEEE Member, His research fields are image professing and intelligence information processing. 
International Journal of $u-$ and e-Service, Science and Technology Vol. 7, No. 6 (2014) 